\title{
The photoaging and fire performance of polypropylene containing melamine phosphate
}

Yuchun Li ${ }^{\text {a,b }}$, Boqiong Xue ${ }^{\mathrm{a}, \mathrm{b}}$, Shuheng Wang ${ }^{\mathrm{a}, \mathrm{b}}$,Jun Sun ${ }^{\mathrm{a}, \mathrm{c}}$, Hongfei Li ${ }^{\mathrm{a}, \mathrm{b}}$, Xiaoyu Gu a , Haiqiao Wang a,*, Sheng Zhang a,b,*

${ }^{a}$ State Key Laboratory of Organic-Inorganic Composites, Beijing University of Chemical Technology, Beijing, 100029, P. R. China

${ }^{\mathrm{b}}$ Key Laboratory of Carbon Fiber and Functional Polymers, Ministry of Education, Beijing University of Chemical Technology, Beijing, 100029, China

${ }^{\mathrm{c}}$ Institute of Textiles and Clothing, the Hong Kong Polytechnic University, Hong Kong, China

*Corresponding authors: Sheng Zhang, zhangsheng@mail.buct.edu.cn. Tel.: +86 (10) 64436820; Haiqiao Wang, wanghaiqiao@mail.buct.edu.cn. Tel.: +86 (10) 64419631 
Supporting Information (SI)

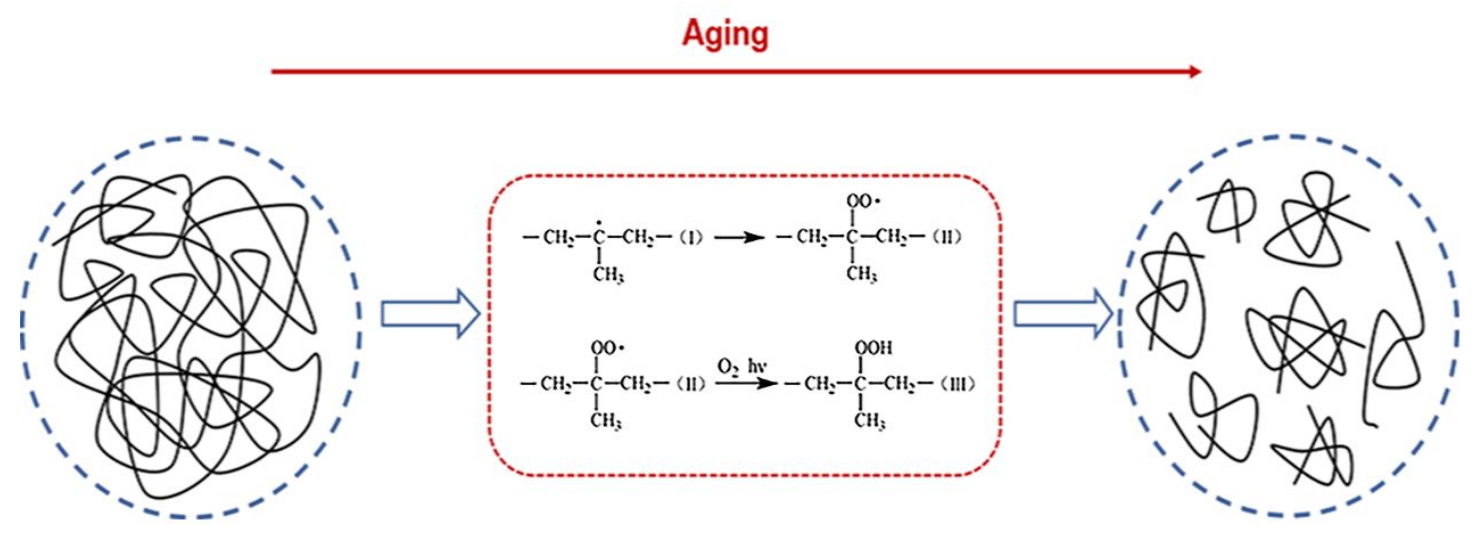

Figure.S1. Photooxidative degradation process of PP.
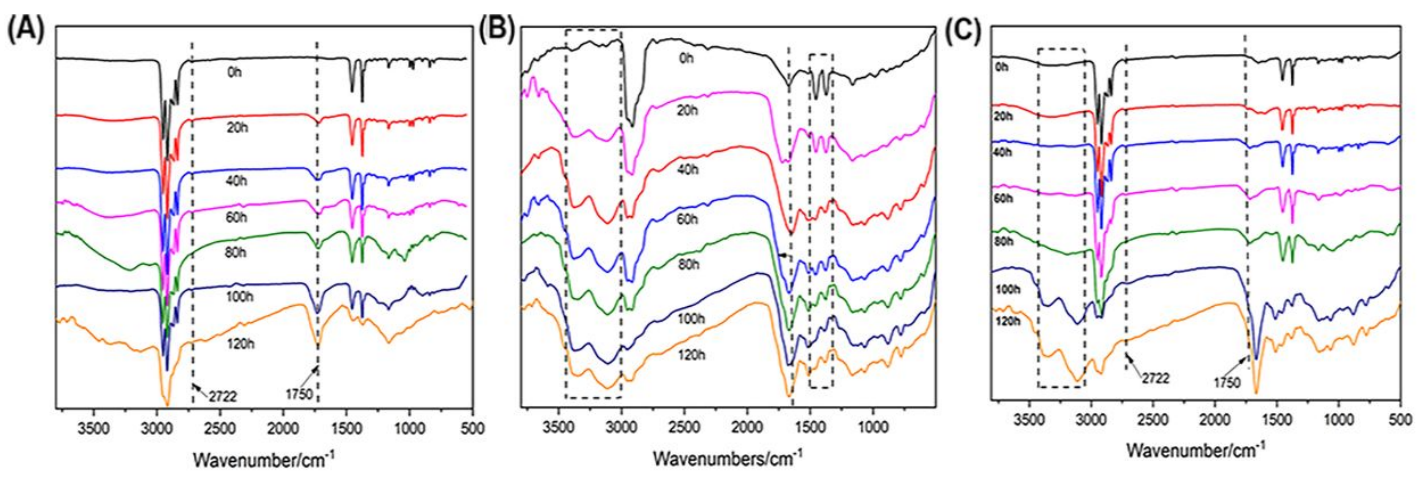

Figure.S2. The FTIR spectra of PP(A), PP/MPP10(B) and PP/MPP20(C) at different irradiation times. 
(A)

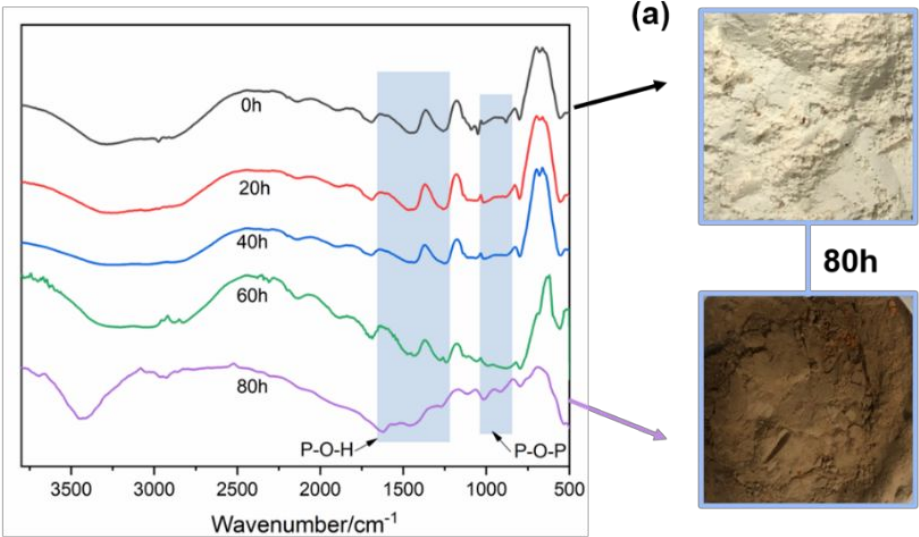

(B)

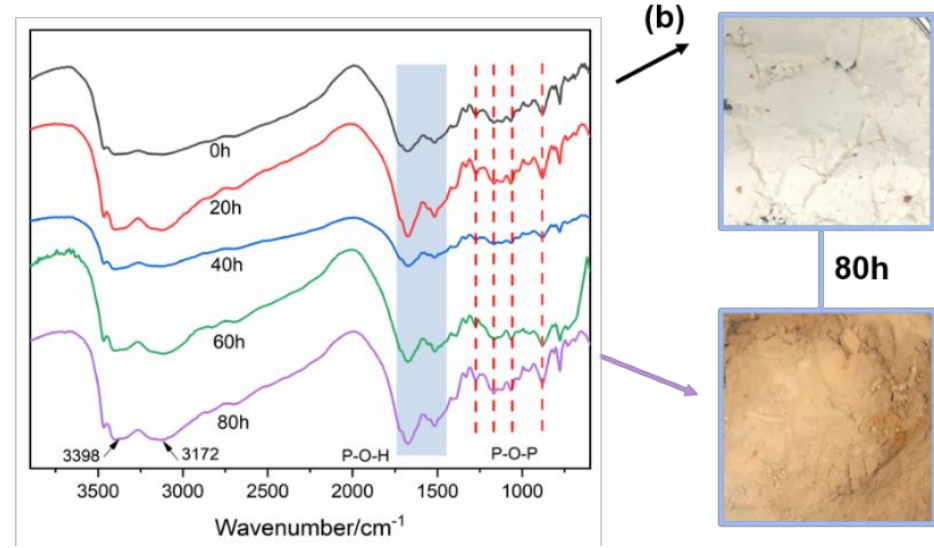

Figure.S3. FTIR spectra of APP(A) and MPP(B) at different irradiation times;

The digital images of APP(a) and MPP(b) before and after $80 \mathrm{~h}$ UVA irradiation. 